\title{
CheXImageNet: a novel architecture for accurate classification of Covid-19 with chest $x$-ray digital images using deep convolutional neural networks
}

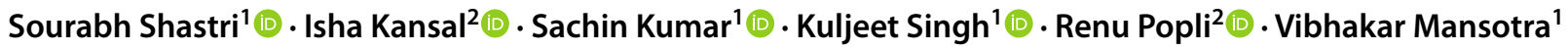

Received: 29 June 2021 / Accepted: 3 December 2021 / Published online: 11 January 2022

(c) IUPESM and Springer-Verlag GmbH Germany, part of Springer Nature 2021

\begin{abstract}
Many countries around the world have been influenced by Covid-19 which is a serious virus as it gets transmitted by human communication. Although, its syndrome is quite similar to the ordinary flu. The critical step involved in Covid-19 is the initial screening or testing of the infected patients. As there are no special detection tools, the demand for such diagnostic tools has been increasing continuously. So, it is eminently admissible to find out positive cases of this disease at the earliest so that the spreading of this dangerous virus can be controlled. Although, some methods for the detection of Covid-19 patients are available, which are performed upon respiratory based samples and among them, a critical approach for treatment is radiologic imaging or X-ray imaging. The latest conclusions obtained from X-ray digital imaging based algorithms and techniques recommend that such type of digital images may consist of significant facts regarding the SARS-CoV-2 virus. The utilization of Deep Neural Networks based methodologies clubbed with digital radiological imaging has been proved useful for accurately identifying this disease. This could also be adjuvant in conquering the problem of dearth of competent physicians in far-flung areas. In this paper, a CheXImageNet model has been introduced for detecting Covid-19 disease by using digital images of Chest X-ray with the help of an openly accessible dataset. Experiments for both binary class and multi-class have been performed in this work for benchmarking the effectiveness of the proposed work. An accuracy of 100\% is reported for both binary classification (having cases of Covid-19 and Normal X-Ray) and classification for three classes (including cases of Covid-19, Normal X-Ray and, cases of Pneumonia disease) respectively.
\end{abstract}

Keywords Coronavirus · Covid-19 · Digital Images X-ray · Deep Neural Network · Image Classification

This article is part of the Topical Collection on COVID-19 Health Technology: Design, Regulation, Management, Assessment.

Sourabh Shastri

sourabhshastri@gmail.com

Isha Kansal

isha.kansal@chitkara.edu.in

Sachin Kumar

skh.sachinkumar@gmail.com

Kuljeet Singh

kuljeetshan94@gmail.com

Renu Popli

renu.popli@chitkara.edu.in

Vibhakar Mansotra

vibhakar20@yahoo.co.in

1 Department of Computer Science \& IT, University of Jammu, 180006 Jammu \& Kashmir, India

2 Chitkara University Institute of Engineering and Technology, Chitkara University, Punjab, India

\section{Introduction}

Coronaviruses are a set of viruses which is harmful to entire human-kind. These contain non-parceled RNA viruses which belong to the family Coronaviridae that are harmful if present in human beings as well as the animals [1,2]. The first known epidemic known as "severe acute respiratory syndrome" happened in 2003. After that, in 2012, 2nd storm of a large epidemic named "middle east respiratory syndrome" happened in Saudi Arabia. The Covid-19 disease started as a storm from Wuhan, the People's Republic of China at the end of December month of 2019. The disease was found till the mid of April month in 2020 [3]. The common symptoms of this virus disease include fever, dry coughing along tiredness. Generally, in most cases, the patient starts feeling difficulty in taking a breath. In some other cases, patients may feel headaches, nausea etc. It gets disseminate from one person to the other one through the 
channel of cough or sneeze droplets by a person who is already infected with the disease [4]. Also, an uninfected one can catch this epidemic virus by even touching those droplets and then touching back to his face, especially the sense organs including eyes, nose, and mouth without washing off their hands. World Health Organization declared this Covid-19 as a public health extremity of international worry in January, 2020. In March of 2020, apart from China, more than 1.1 million cases were discovered and over four thousand deaths took place in more than 110 countries. World Health Organization (WHO) declared this Covid-19 situation as a pandemic [5]. Globally, many people from the research community of different fields such as the medical field, clinical field, and the field of artificial intelligence are trying hard to form some plans to fight against Covid-19.

Covid-19 is currently the biggest challenge to the entire human race regarding health, economic, and survival issues. We are in urgent need to find solutions, treatment, and develop vaccines to fight against it. One of the challenges in developing vaccines for Covid-19 is that we have not yet understood this virus. Shastri et al. [6] proposed the variants of long as well as short-termed retention such as stacked, dual-directional, and Convolutional based neural networks which are now used to calculate the Covid-19 cases. The reaction called Reverse Transcription Polymerase Chain Reaction is the most common technique of testing the true cases of Covid-19. The given test is applied on the respiratory organ's samples of the diseased person, and the outcomes of this test are being generated very shortly just within a few hours or in few cases, it may take two days to do. In addition to this, Antibodies are utilized to identify Covid-19 disease, in which the blood samples of the infected patients are being used to identify this. But, some medical professionals also utilize the Chest X-ray scans of the patients to specify the condition of the lungs.

There are a lot of medical diagnosis processes for example X-ray, CT which are very helpful in this pandemic [7-9]. Clinically, the detection of nucleic acid is normal but the accessibility, steadiness, and production of test kits of nucleic acid are one of the main problems. Having large demand for test kits in different parts of the world results in a slow pace of new corona pneumonia screening [10]. Due to less availability of test kits, a lot of the patients with this new corona pneumonia are not being able to get admitted to hospital which leads to widespread of this virus. At this point in time, testing is the only available way to stop this widespread. As the industry growing in healthcare is successful to manage and provide low-cost kits, so the process becomes easy and they are successful in accelerating the work and create a wide gap between demand and supply. One another way is medical imaging examinations, which can help detection of disease conveniently and quickly which has been used to diagnose Covid-19 worldwide [11]. Medical images like X-rays do have the uncommon superiority of lightness, fast, and availability in the testing of Covid19. Normal X-ray machines may be utilized in hospitals where CT scanning machines are present in less amount. These images can be enhanced by using digital image processing techniques including $[12,13]$ etc. Mainly, the easily accessible X-ray examination is even more beneficial for quick testing on very large scale $[14,15]$. Artificial Intelligence with digital image processing is an eminent way for differentiating the X-ray images of normal and infected patients. This also provides great accuracy in classification. It is likely to improvise the accuracy of Covid-19 disease identification and to curtail its disperse mainly in source-restraint areas where medical specialists and radiologists professionals may not be available for initial examination and supervision of patients of Covid19 disease [16].

In this paper, a CheXImageNet model has been introduced for detecting Covid-19 disease by using digital images of Chest X-ray with the help of an openly accessible dataset. Experiments for both binary class and multi-class have been performed in this work for benchmarking the effectiveness of the proposed work. An accuracy of $100 \%$ is reported for both binary classification (having cases of Covid-19 and Normal X-Ray) and classification for three classes (including cases of Covid-19, Normal X-Ray and cases of Pneumonia disease) respectively. The proposed work can be a competent channel of examination for predicting Covid-19 disease in the human body and can help radiologists and medical professionals.

The rest of the paper is organized as follows: Section 2 represents the review of recent scholarly works that are related to the present study. Section 3 describes the research methodology with data description and methods used in this study along with the algorithm of the proposed model. Section 4 contains the proposed experimental setup. Section 5 contains the discussion of various Covid-19 affected educational parameters along with Covid-19 and human body cell interaction. Section 6 gives the conclusion and future work of the study.

\section{Literature survey}

In the past few years, with an immense advancement in science and technology, the applications of Computer Vision based techniques and Deep Learning approaches are continuously being utilized to uncover various ailments in the body automatically like sores in various 
body parts, detection of various types of tumors in the human body and aggregation in body parts including lungs, chest, skull or even brain. Further diseases like the categorization of images having diabetic disease, prostate diagnosis, nodule categorization, skin sores categorization, investigation of the myocardium in coronary CT angiography and tracking etc. [17-20]. In current times, Chest CT scans and digital X-ray image testing have been used for identifying Covid-19 in a human body. But the utilization of vision based computing and deep learning methodologies can also play a huge role in identifying the presence of coronavirus. With Covid-19 becoming a widespread health problem, the research community and scientists have obtained great results using automated vision and Deep Learning based mechanisms. In [21], Yang et al. performed an analytic analysis of CT scans and categorized the suspected cases into various classes for diagnosis and treatment. In [22], Wang et al. had used an ingenious $\mathrm{CNN}$ to classify and predict Coronavirus using lung CT scans. Covid-Net uses insubstantial residual projection expansion projection extension design pattern to investigate quantitative and qualitative analysis [23].

In another research study, pre-trained ResNet50, InceptionV3, and Inception ResNetV2 models have been used with transfer learning techniques to classify Chest X-ray images as normal and Covid-19 classes [37]. In [24], the authors present COVNet to predict Covid-19 from CT scans that have been divided using U-net [25]. In [26], authors brought up a VGG-16 Network-based Faster Regions with Convolutional Neural Networks framework to find Covid-19 patients. Li et al. [27] reported a discriminative cost-sensitive learning solution, which integrates advantages emerging from fine-grained categorization and cost-sensitive learning. In [28], authors suggested the utilization of transfer learning along with a descriptive model to identify the disease of Coronavirus. Authors in [29], introduced a DarkNet model as a classifier. The work in [30] varies from the above-mentioned studies in terms of the research methodology, as they first extracted the deep features through an extensive convolutional network (ResNet50) and then categorized the Covid-19 cases derived from the remaining Chest X-ray images using a support vector machine. Their suggested model is comprised of seventeen layers of convolutional neural networks with varying filters.

In [31], authors presented an illustratable Artificial Intelligence framework evaluated by professional radiologists based on the amount of focus attention maps put on diagnostically relevant image portions. Most of the datasets have limited samples of the positive cases of Covid-19, which increases the challenge for impartial learning. Taking this into account, [32] presented the random oversampling and weighted class loss functional approach for unbiased fine-tuned learning (transfer learning) in various state-of-the-art deep learning approaches. In [33], authors proposed deep learning (DL) based dual-tasks network, named FaNet, which performs both diagnosis and serious assessments for Covid-19 conditional to the combination of 3D CT imaging and clinical symptoms. Based on the enhancement of the classical visual geometry group network with the convolutional Covid block, an efficient and effective screening model was proposed by [34] to diagnose and differentiate patients with the Covid-19 from those with pneumonia and other healthy people through radiography.

In [35], the authors brought up a Deep Convolutional Neural Network method for fast and reliable identification of Covid-19 infection cases from the sufferer's Chest X-ray images. In [36], authors created a dataset consisting of publicly available X-ray images from patients with confirmed Covid-19 disease and common bacterial pneumonia and also the healthy individuals. Transfer learning was employed, to mitigate a small number of samples transferring knowledge extracted by pre-trained models to the model to be trained. In [37], a model for Covid19 detection was proposed using raw Chest X-ray which could provide accurate diagnostics for binary class classification (Covid-19 cases and cases with no findings) and multi-class classification (Covid-19 cases, cases with no findings and, cases of Pneumonia). This work implemented seventeen layers of convolutional with varied filtering on each layer. Training techniques were introduced in [38] which helped to learn the network better in the case where the dataset is unbalanced (for example, very few cases of Covid-19 but more cases from normal or Pneumonia). They also presented a neural network that is a combination of the Xception and ResNet50V2 networks.

\section{Materials and methods}

In this section, the description of the dataset and the research methodology has been given in detail:

\subsection{Dataset description}

Dataset is taken from 4 open-source data sources. The link is given in table from where the data for the experiment was taken.

In Fig. 1, the distribution of data is represented in form of a pie chart for binary class (Covid-19 cases and Normal 
Fig. 1 Data distribution chart for (a) Binary class and (b) Multi-class experiment

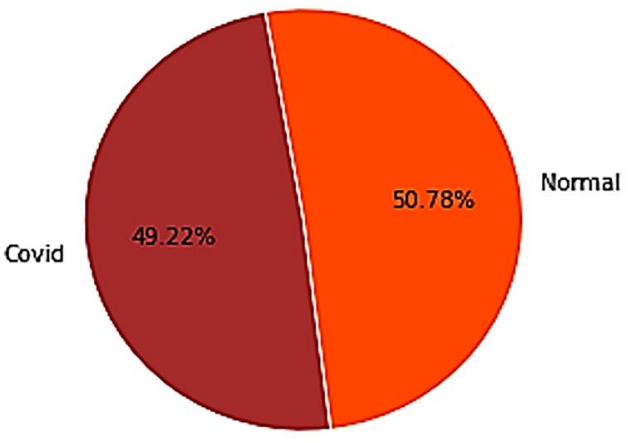

(a)

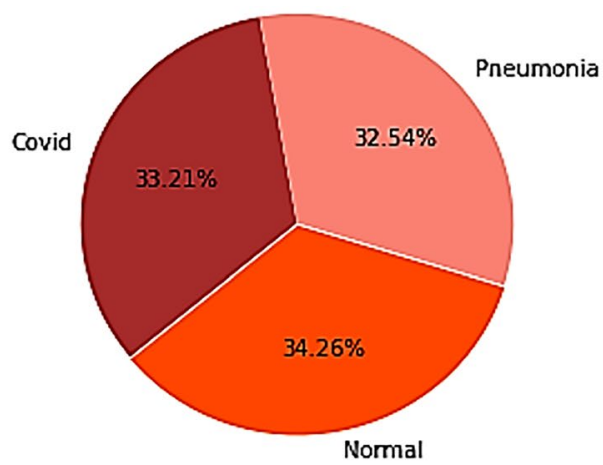

(b) cases) and also for multi-class (Covid-19 cases, Normal cases, and Pneumonia cases).

In Table 1, three types of image data have been categorized including cases of Covid-19, cases of Normal persons, cases showing Pneumonia images, and their references are given in subsequent rows. Also, the number of figures in each category is specified. The actual images for all these three categories are shown in Fig. 2.

\subsection{Research methodology}

This section contains details about the parameter tuning and the proposed CheXImageNet Architecture in detail.

Figure 3 shows the flow of the proposed work. Firstly, the image dataset is collected as per description in Table 1 . The dataset is pre-processed including merging of the datasets, resizing the images to $256 \times 256$. Finally shuffling the data and converting all the images from gray scale to $R G B$ images. After the data pre-processing step, data is split into training (80\%) and testing (20\%). The proposed CheXImageNet is trained on the dataset. Then the proposed model is evaluated by using various parameters including Accuracy, Precision, Specificity, Sensitivity and F1-Score. Finally, after getting the desired results, the digital images are categorized into two different categories (Normal cases and Covid-19 cases images) for binary class classification and 3 categories (Covid-19 cases, Normal cases and, Images of Pneumonia cases) for classification in multi-class.

\subsubsection{Parameter tuning}

The images in datasets are not of the same resolution. So, all the images have been made to a common size of $256 \times 256$ pixels. The given dataset has been split randomly into $80 \%$ and $20 \%$ for training and testing.

To train the model, 100 epochs were conducted to avoid the problem of overfitting with a batch size of 25 . To compile a deep learning model, an optimizer is required. Optimizers helps to get faster results. The proposed model is compiled with the adam optimizer for $1 \mathrm{e}-3$, and 0.8 as the initial learning rate, and momentum respectively. The main motive of using adam optimizer is that it requires little memory and computationally it is very efficient. Whereas the learning rate determines the rate of learning of the deep learning model that decides the number of moves required to minimize the value of loss function, and the momentum is used to improves both model training speed and accuracy. To determine the output of a node like 1 or 0 , a function is used named activation function. An activation function is added to help the neural network to learn complex patterns of X-ray image data. The activation function used for the experiment is Leaky ReLU.

\subsubsection{Proposed CheXImageNet architecture}

The flow blueprint of the proposed work is shown in Fig. 3. The procedure starts with collecting the X-ray

Table 1 Dataset description

\begin{tabular}{|c|c|c|}
\hline Class & Number of Figures & References \\
\hline Covid & 347 & $\begin{array}{l}\text { https://www.kaggle.com/nabeelsajid917/covid-19-x-ray-10000-images } \\
\text { https://www.kaggle.com/tarandee97/covid19-normal-posteroanteriorpa-xrays }\end{array}$ \\
\hline Normal & 358 & https://www.kaggle.com/pranavraikokte/covid19-image-dataset \\
\hline Pneumonia & 340 & https://www.kaggle.com/paultimothymooney/chest-xray-pneumonia \\
\hline
\end{tabular}




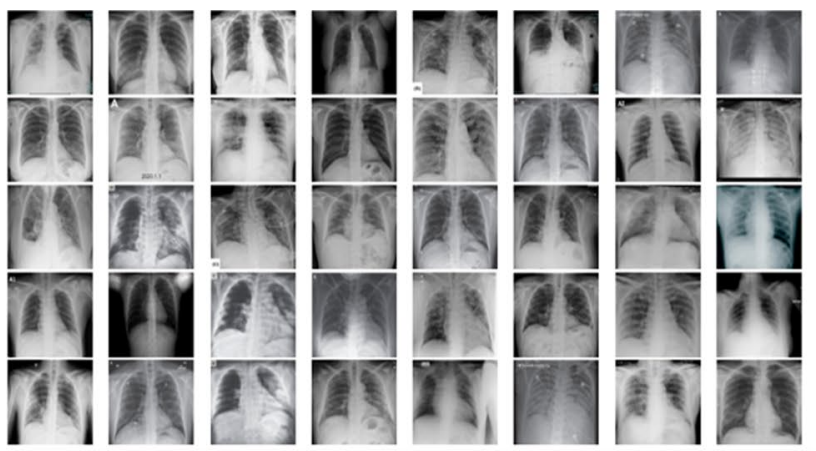

(a)

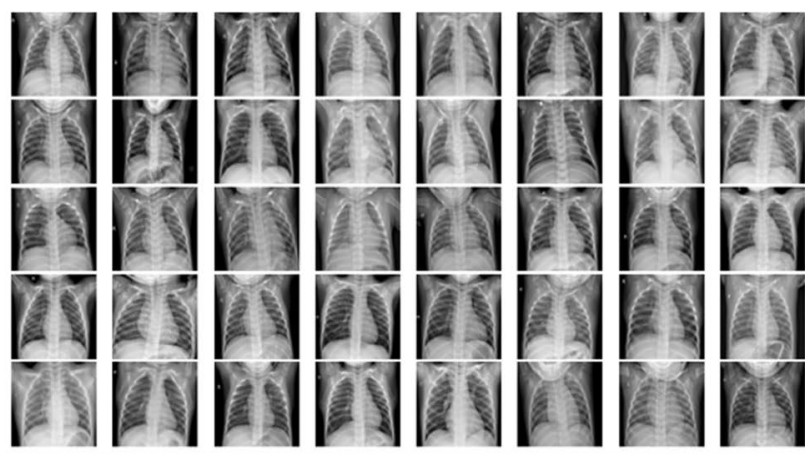

(b)

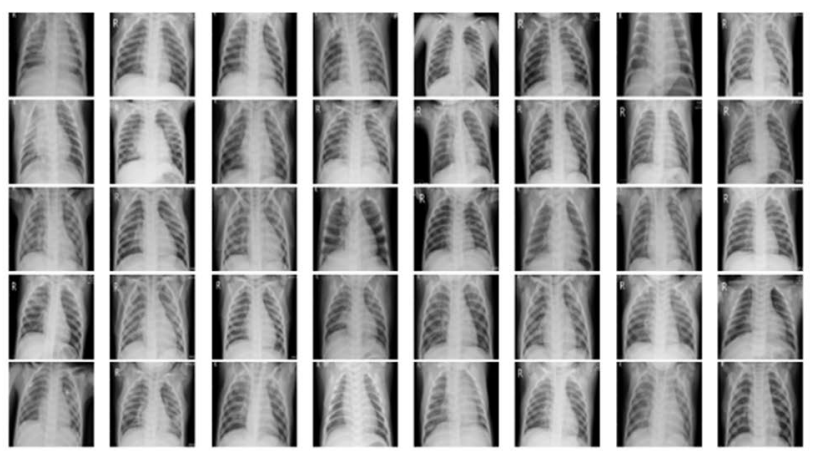

(c)

Fig. 2 Random digital images from different datasets: (a) Digital images of chest X-Ray showing Covid-19, (b) Showing cases having Normal images and (c) showing cases having Pneumonia images

image dataset from open sources followed by the data prepreparing step. In this step, different datasets are merged to form the larger one and then the images in the dataset are resized to a fixed size of $256 \times 256$. Finally, the images are shuffled and the images are converted to RGB (True Color) images. After the data gets pre-processed, it is split into training data images ( $80 \%)$ and testing data images (20\%). After this process, the proposed model comes into the picture which is a "CheXImageNet". The model is then applied to training data to form the trained model and then tested on test data.

In the proposed work, the model is evaluated with various standard factors including the Accuracy of the model, Precision attained, Specificity obtained, Sensitivity factor and, finally the F1-Score. After the proposed model gets evaluated, the data can be classified into Covid-19, Normal and, Pneumonia patient categories.

CheXImageNet: "Che" stands for "Chest", "XImage" stands for "X-ray images" and the word "Net" means "Deep Neural Network". The whole meaning of the word is the deep neural network architecture for categorization of Covid-19 infected cases by utilizing digital images of Chest X-ray. Our proposed architecture using a deep neural network named CheXImageNet have some layers and filters. There are 3 convolutional layers, 4 batch normalization layers, 3 max pooling layers, 7 LeakyReLU layers, 5 dense layers, 1 flatten layer, and 4 dropout layers. Each convolutional layer is followed by batch normalization and LeakyReLU operation and then passed to the max pooling layer. LeakyReLu is utilized as a function for activation in the CheXImageNet architecture. The kernel size used in the proposed architecture are $2 \times 2$ and $3 \times 3$. The filters are continuously increased to the number such as $32,64,256$. This model ends with the softmax activation function layer that produces the outputs. The proposed architecture has been shown in Fig. 4.

\section{Experiment evaluation and results}

In this section, experiments performed by the proposed technique and the results are shown in details.

\subsection{Implementation environment}

The experimental work has been carried out in Jupyter notebook provided by google's collaboratory environment 


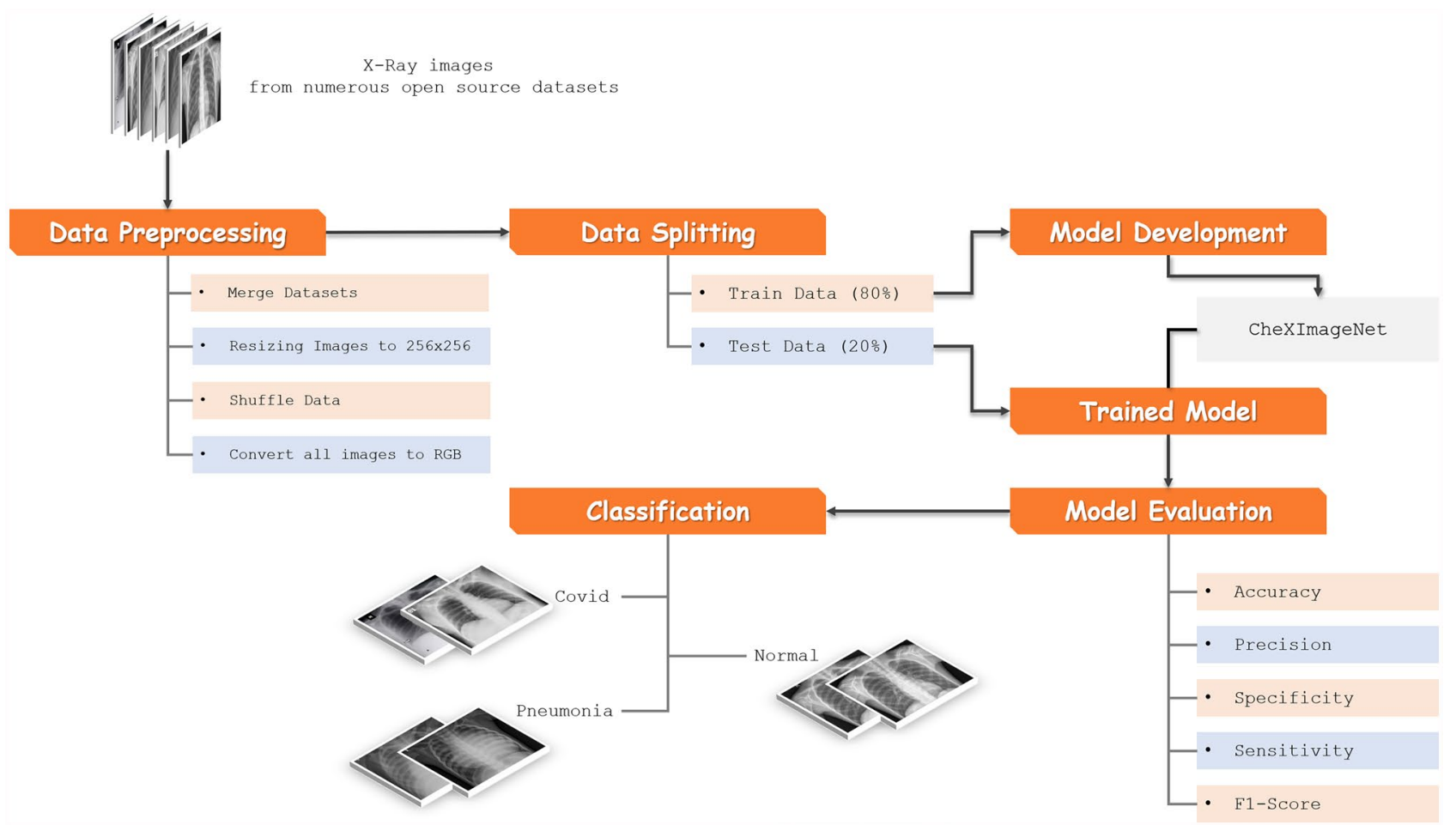

Fig. 3 Flow diagram of proposed classification framework

which is an online platform for training the deep learning model(s). It provides the facility of processing the data on the NVIDIA Tesla K80 GPU with 12 GB of RAM. Python 3 version has been used in the experiment for implementing the proposed algorithm. The main open-source libraries used in experiment are keras, numpy, scikit, pandas, tensorflow, and matplotlib.

\subsection{Performance evaluation and results}

\subsubsection{Model description (binary class experiment)}

In this section, the different layers of the model their shapes, and the parameters for the binary class experiment are shown.
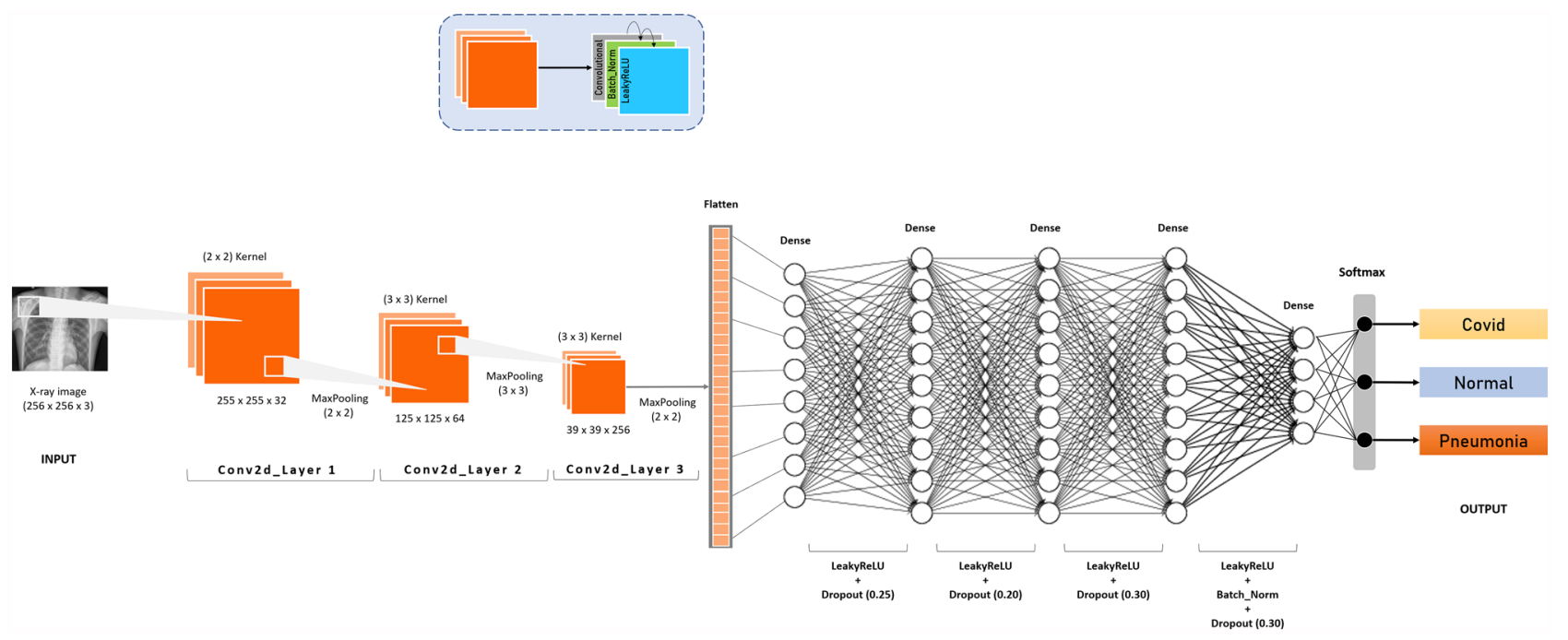

Fig. 4 Proposed CheXImageNet architecture 
Table 2 Model parameters

\begin{tabular}{|c|c|c|c|c|c|}
\hline \multicolumn{3}{|c|}{ Parameters for Binary Class Experiment } & \multicolumn{3}{|c|}{ Parameters for Multi Class Experiment } \\
\hline \multicolumn{3}{|l|}{ Epoch $=100$} & \multicolumn{3}{|l|}{ Epoch $=100$} \\
\hline \multicolumn{3}{|l|}{ Batch $=25$} & \multicolumn{3}{|l|}{ Batch $=25$} \\
\hline \multicolumn{3}{|l|}{ Optimizer $=$ Adamax } & \multicolumn{3}{|l|}{ Optimizer $=$ Adamax } \\
\hline \multicolumn{3}{|l|}{ Number of Classes $=2$} & \multicolumn{3}{|l|}{ Number of Classes $=3$} \\
\hline \multicolumn{3}{|l|}{ Model: "sequential" } & \multicolumn{3}{|l|}{ Model: "sequential" } \\
\hline Layer (type) & Output Shape & Param \# & Layer (type) & Output Shape & Param \# \\
\hline conv2d (Conv2D) & $(255,255,32)$ & 416 & conv2d_24 (Conv2D) & $(255,255,32)$ & 416 \\
\hline batch_normalization & $(255,255,32)$ & 128 & batch_normalization_14 & $(255,255,32)$ & 128 \\
\hline leaky_re_lu & $(255,255,32)$ & 0 & leaky_re_lu_32 & $(255,255,32)$ & 0 \\
\hline max_pooling2d & $(127,127,32)$ & 0 & max_pooling2d_24 & $(127,127,32)$ & 0 \\
\hline conv2d_1 & $(125,125,64)$ & 18496 & conv2d_25 & $(125,125,64)$ & 18496 \\
\hline batch_normalization_1 & $(125,125,64)$ & 256 & batch_normalization_15 & $(125,125,64)$ & 256 \\
\hline leaky_re_lu_1 & $(125,125,64)$ & 0 & leaky_re_lu_33 & $(125,125,64)$ & 0 \\
\hline max_pooling2d_1 & $(41,41,64)$ & 0 & max_pooling2d_25 & $(41,41,64)$ & 0 \\
\hline conv2d_2 & $(39,39,256)$ & 147712 & conv2d_26 & $(39,39,256)$ & 147712 \\
\hline batch_normalization_2 & $(39,39,256)$ & 1024 & batch_normalization_16 & $(39,39,256)$ & 1024 \\
\hline leaky_re_lu_2 & $(39,39,256)$ & 0 & leaky_re_lu_34 & $(39,39,256)$ & 0 \\
\hline $\max \_$pooling2d_2 & $(19,19,256)$ & 0 & max_pooling2d_26 & $(19,19,256)$ & 0 \\
\hline flatten & (92416) & 0 & flatten_8 & (92416) & 0 \\
\hline dense & $(256)$ & 23658752 & dense_40 & $(256)$ & 23658752 \\
\hline leaky_re_lu_3 & $(256)$ & 0 & leaky_re_lu_35 & $(256)$ & 0 \\
\hline dropout & $(256)$ & 0 & dropout_32 & $(256)$ & 0 \\
\hline dense_1 & $(128)$ & 32896 & dense_41 & $(128)$ & 32896 \\
\hline leaky_re_lu_4 & $(128)$ & 0 & leaky_re_lu_36 & $(128)$ & 0 \\
\hline dropout_1 & $(128)$ & 0 & dropout_33 & $(128)$ & 0 \\
\hline dense_2 & $(64)$ & 8256 & dense_42 & (64) & 8256 \\
\hline leaky_re_lu_5 & $(64)$ & 0 & leaky_re_lu_37 & $(64)$ & 0 \\
\hline dropout_2 & $(64)$ & 0 & dropout_34 & (64) & 0 \\
\hline dense_3 & $(32)$ & 2080 & dense_43 & $(32)$ & 2080 \\
\hline leaky_re_lu_6 & $(32)$ & 0 & leaky_re_lu_38 & (32) & 0 \\
\hline batch_normalization_3 & $(32)$ & 128 & batch_normalization_17 & $(32)$ & 128 \\
\hline dropout_3 & $(32)$ & 0 & dropout_35 & $(32)$ & 0 \\
\hline dense_4 & (2) & 66 & dense_44 & (3) & 99 \\
\hline Total: $23,870,210$ & & & Total: $23,870,243$ & & \\
\hline Trainable: $23,869,442$ & & & Trainable: $23,869,475$ & & \\
\hline Non-trainable: 768 & & & Non-trainable: 768 & & \\
\hline
\end{tabular}

\subsubsection{Model description (multi-class experiment)}

In this section, the different layers of the model their shapes, and the parameters for the multi-class experiment are shown (See Table 2).

\subsection{Visualization}

In this section, the heatmap and the attention maps obtained by the proposed technique have been shown in Figs. 5 and 6 respectively.

\subsection{Comparative analysis}

The authors have performed two experiments, one for Covid-19 detection as a binary class (Covid-19, Normal) problem and the second experiment that is multi-class (Covid-19, Normal, Pneumonia) problem. Both binary and multi-class models are trained for 100 epochs where the size of the batch is set to 25 . The X-ray images of size $256 \times 256$ are used for training and testing the models. An early stopping method is used to end learning, to avoid overfitting. The models are compiled with the 


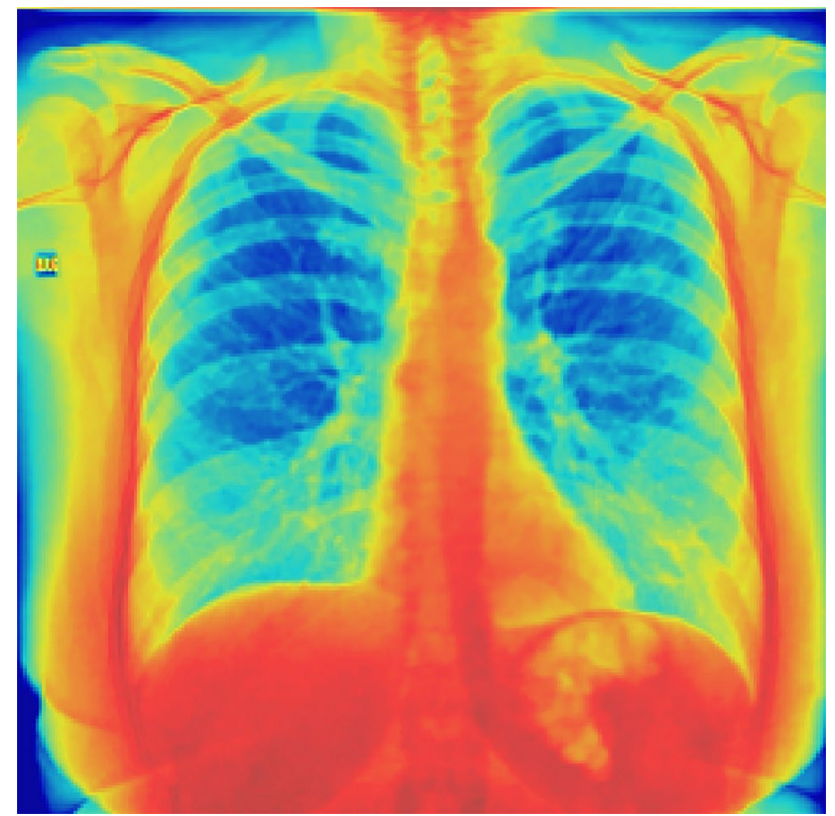

Fig. 5 Heat map of resized X-ray image $(256 \times 256)$

adamax optimizer, where 0.00001 , and 0.9 are used as an initial learning rate, and momentum respectively. In Table 3, the proposed work has been compared to other ones in terms of Accuracy (\%) for both binary class and multi-class. Our work has been compared with eight existing Techniques including Ioannis [28], Tulin [29], Wang [23], Sethy [30], Zheng [9], Wang [7], Xu [8], Ali [34]. These techniques consider 224 cases of Covid-19 (+) 700 cases of pneumonia 504 healthy cases, 125 Covid-19(+) cases 500 cases of pneumonia 500 cases with no findings, 53 cases of Covid-19(+) 5526 cases of Covid-19(-) 8066 cases of healthy, 127 facts of Covid-19(+) 127 facts of normal 127 facts of pneumonia, 313 cases of Covid-19 (+) 229 cases of Covid-19(), 195 cases of Covid-19(+) 258 cases of Covid-19(-), 219 facts of Covid-19(+) 224 facts of pneumonia 175 cases of healthy, 310 facts of Covid-19(+) 654 facts of healthy 864 cases of pneumonia respectively. The proposed study considers 347 facts of Covid-19 358 facts of normal and also 340 facts of pneumonia. As it can be seen in Table, the proposed technique outperforms all the techniques by achieving $100 \%$ Accuracy. The confusion matrix for both experiments is presented in Fig. 7. Table 4 illustrates the results of our proposed technique in terms of performance metrics. From Table 4, the overall accuracy of both binary and multi-class experiment is $100 \%$. Moreover, the precision, sensitivity, specificity, and f1-score for both experiments are also $100 \%$. Figures 8 and 9 present the classification accuracy and loss curves in a train and the test set during the training of the model for binary class experiment and multi-class experiment.
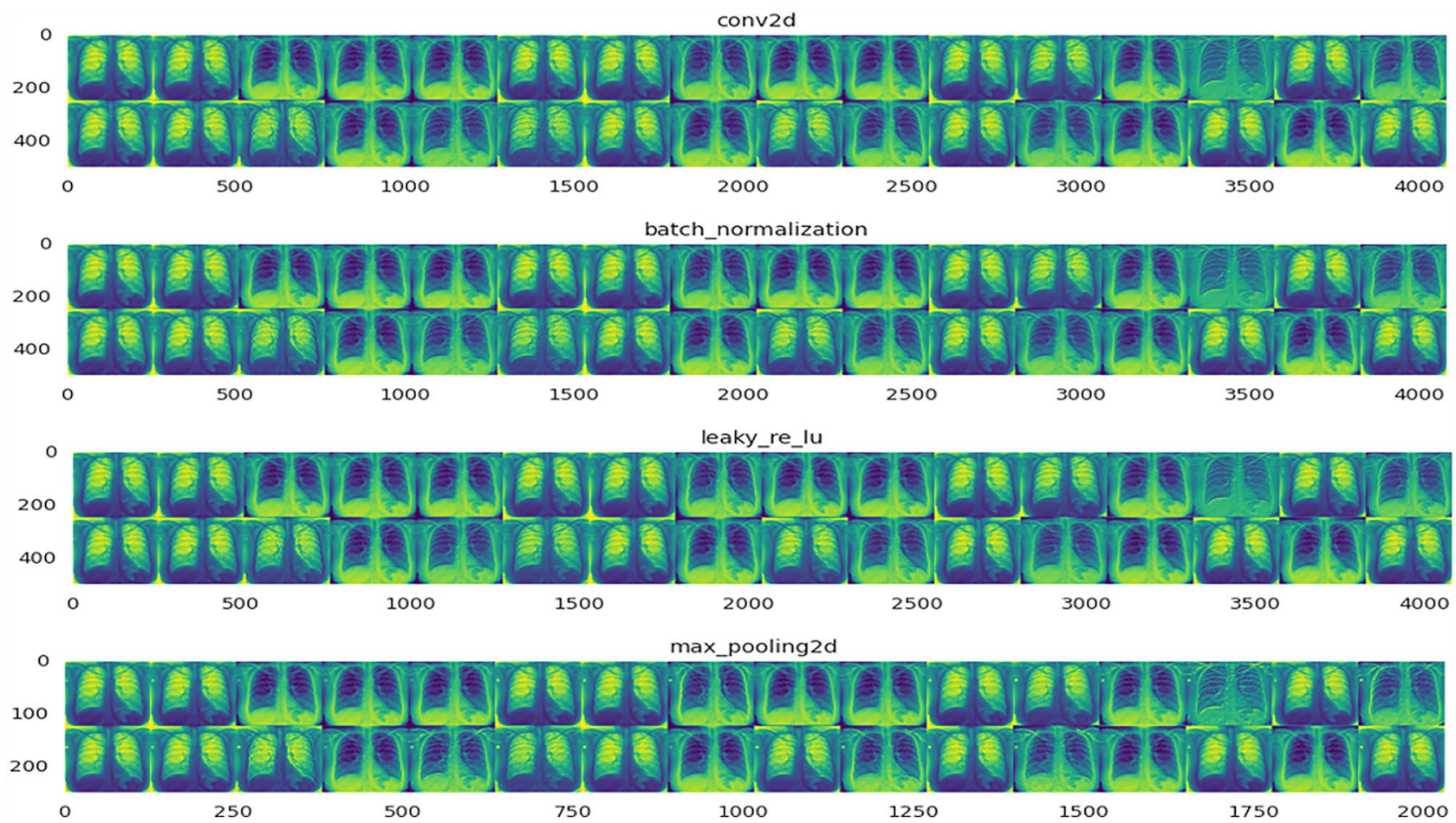

Fig. 6 Attention map of Conv2d_Layer 1 
Table 3 Comparative analysis between the suggested technique and other techniques

\begin{tabular}{|c|c|c|c|c|}
\hline Technique & Image Types & Technique Used & $\begin{array}{l}\text { Accuracy for (Two- } \\
\text { Classes) }(\%)\end{array}$ & $\begin{array}{l}\text { Accuracy for } \\
\text { Three-Classes } \\
(\%)\end{array}$ \\
\hline Zheng et al. [9] technique & Digital image of CT & UNet+Three D Deep Network & 90.8 & NA \\
\hline Ioannis et al. [28] technique & Digital image of X-ray & VGG-19 & NA & 93.48 \\
\hline Wang and Wong technique [23] & Digital image of X-ray & Covid-Net & NA & 92.4 \\
\hline Sethy and Behra technique [30] & Digital image of X-ray & ResNet50+ SVM & NA & 95.33 \\
\hline Wang et al. [7] technique & Digital image of CT & M-Inception & 82.9 & NA \\
\hline $\mathrm{Xu}$ et al. [8] technique & Digital image of CT & ResNet + Location Attention & NA & 86.7 \\
\hline Ali et al. [34] technique & Digital image of CT & DRE-Net & 98.86 & 95.51 \\
\hline Tulin et al. [29] technique & Digital image of X-ray & DarkCovidNet & 98.08 & 87.02 \\
\hline Our technique & Digital image of X-ray & CheXImageNet & 100 & 100 \\
\hline
\end{tabular}

\section{Discussion}

The emanation of Covid-19 is a dangerous gasping disease that has expeditiously disseminated across the blob creating colossal public health issues. This disease has afflicted the lives of lots in many ways in distinct geological areas worldwide. Above the immediate hazard to the human body, cutback in employment, vacillation etc. Education is the hypersensitive area that is afflicted due to Covid-19 excessively. At this time of adversity, different national level and university based exams are delayed. This in turn has affected many teaching-learning programs. Therefore, many educational organizations have encouraged the system of online education by introducing virtual or online classes by making sure that the learning process remains valid for the students across the world as the best practical remedy for the continuation of teaching-learning processes. Daily routines of older people, their support and care, their ability to remain connected socially are also getting affected due to Covid-19. They are being forced by the requirements of the day to spend more time at home, avoiding physical contact with their family members, relatives, friends and, other colleagues. During the pandemic, pregnant women and newborns represent a susceptible community. It is a well-known fact that conceived ladies are at much higher risk for serious sickness with viruses like Covid-19 and another vigorous respiratory. The dissemination of the virus has mainly centered on the global nature of world-level tourism and how the region may be influenced by this virus. As per the discussion in the above paragraph, Covid-19 has affected human lives in various ways. Therefore it has become challenging to detect this disease at initial stages without affecting human health. In the proposed work, a technique for Covid-19 Chest X-ray image classification has been proposed which provides $100 \%$ Accuracy.

WHO recommended RT-PCR test for Covid-19. But, the test is confined to a limited number of laboratories. The short accuracy of the RT-PCR test encourages health experts and researchers to set up more ways of diagnosis. The radiological study for diagnosing infectious diseases such as arthritis, tuberculosis, osteoporosis, pneumonia, etc. for many decades. But the manual reading of X-ray images is a slow-moving task. It is difficult for health experts to guarantee an immediate response in the present situation of the global pandemic. No doubt, the RT-PCR test method to detect Covid-19 is still important. However, there are also some proved
Table 4 Table showing the performance of the proposed technique in classification

\begin{tabular}{lllllll}
\hline Experiment Type & Label & $\begin{array}{l}\text { Obtained } \\
\text { Precision }\end{array}$ & $\begin{array}{l}\text { Obtained } \\
\text { Sensitivity }\end{array}$ & $\begin{array}{l}\text { Obtained } \\
\text { Specificity }\end{array}$ & $\begin{array}{l}\text { Obtained } \\
\text { F1-Score }\end{array}$ & $\begin{array}{l}\text { Overall } \\
\text { Accuracy }\end{array}$ \\
\hline Binary class & Covid-19 & 100 & 100 & 100 & 100 & $\mathbf{1 0 0 \%}$ \\
& Normal & 100 & 100 & 100 & 100 & \\
Multi-class & Covid-19 & 100 & 100 & 100 & 100 & $\mathbf{1 0 0 \%}$ \\
& Normal & 100 & 100 & 100 & 100 & \\
& Pneumonia & 100 & 100 & 100 & 100 & \\
\hline
\end{tabular}




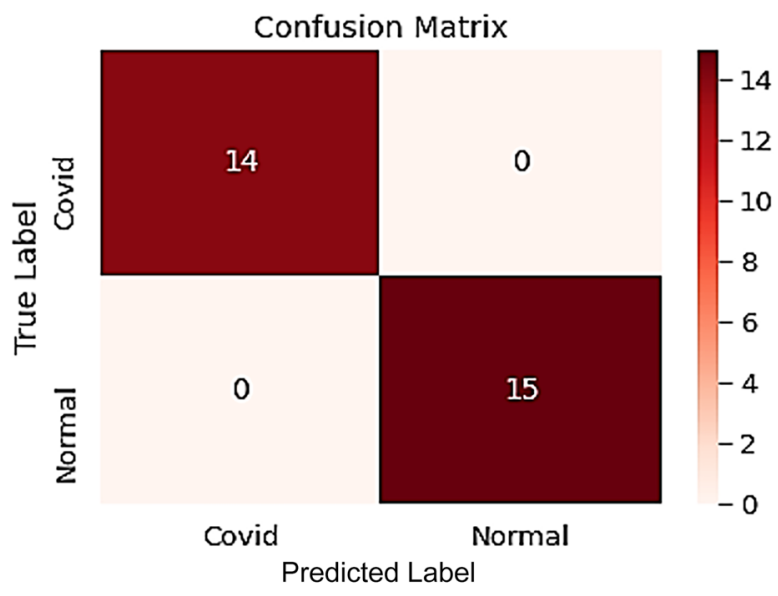

(a)

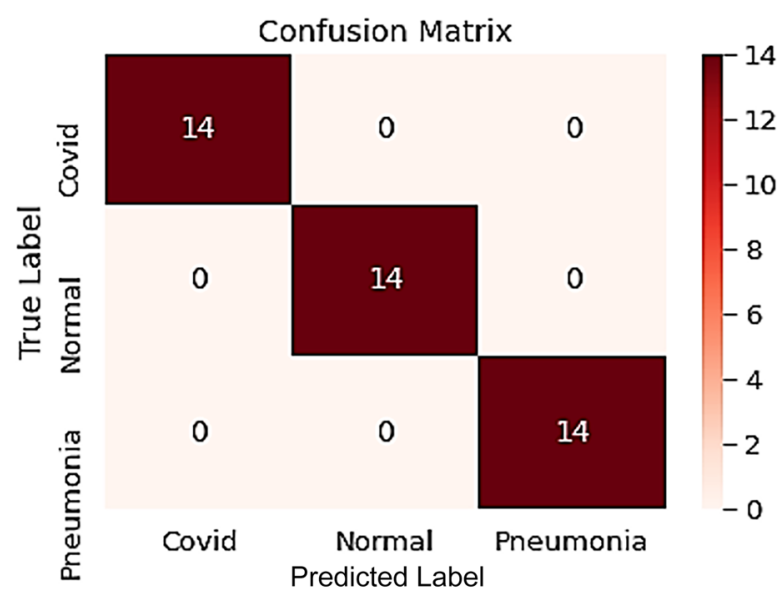

(b)

Fig. 7 Confusion matrix for (a) Binary class experiment and (b) Multi-class experiment

the weakness of the RT-PCR test method such as delay in response time, and the possibility for collecting the specimens in mistaken localizations, etc. The proposed model can identify Covid-19 affected person and even pneumonia person without any human interference at an economical cost with higher accuracy. Therefore, we believe this proposed model might be of assistance for health professionals in the early diagnosis of Covid-19.

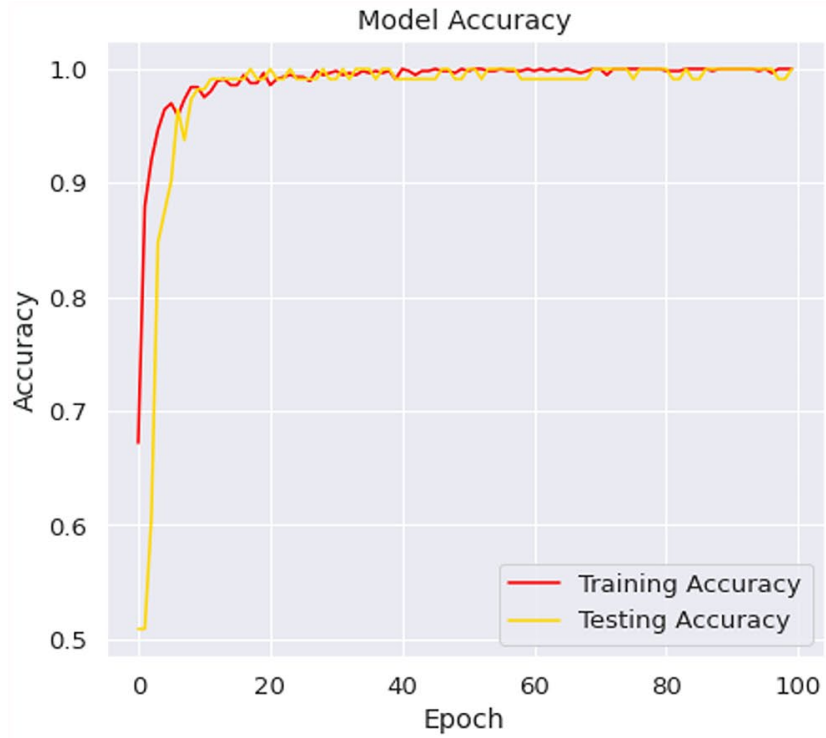

(a)

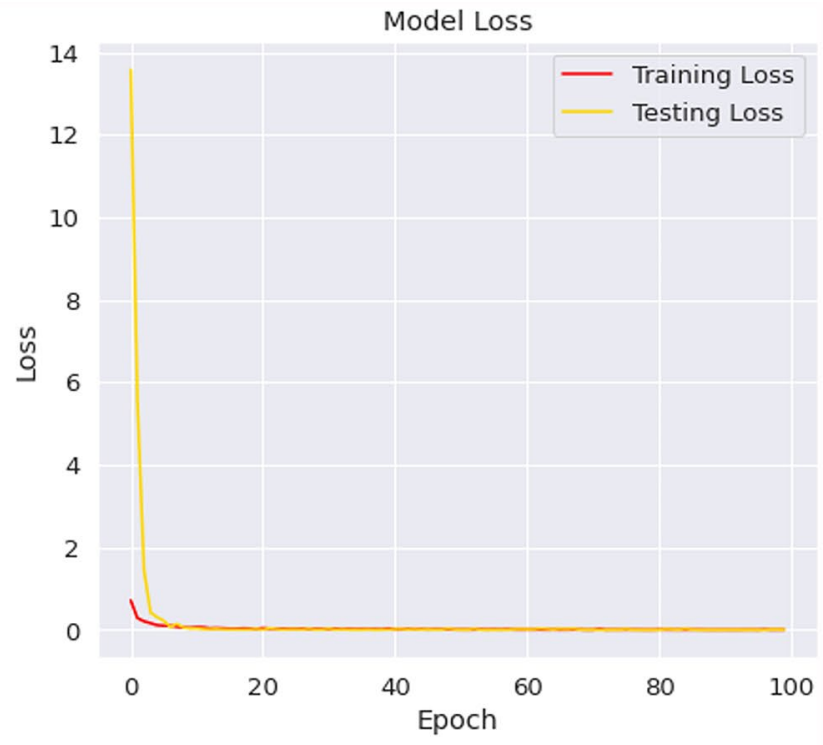

(b)

Fig. 8 Training and Testing (a) Accuracy (b) Loss for binary class experiment 


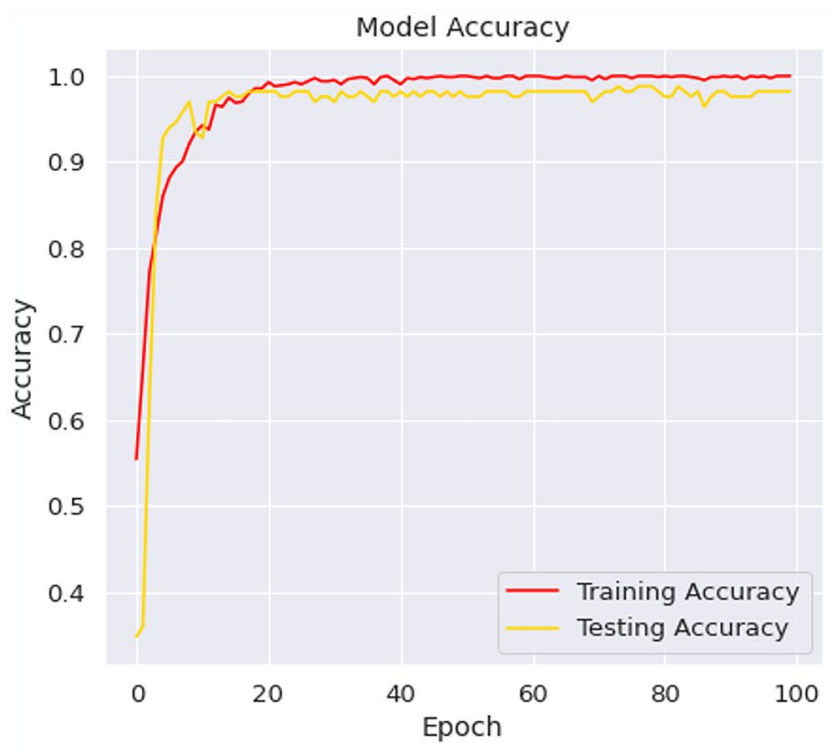

(a)

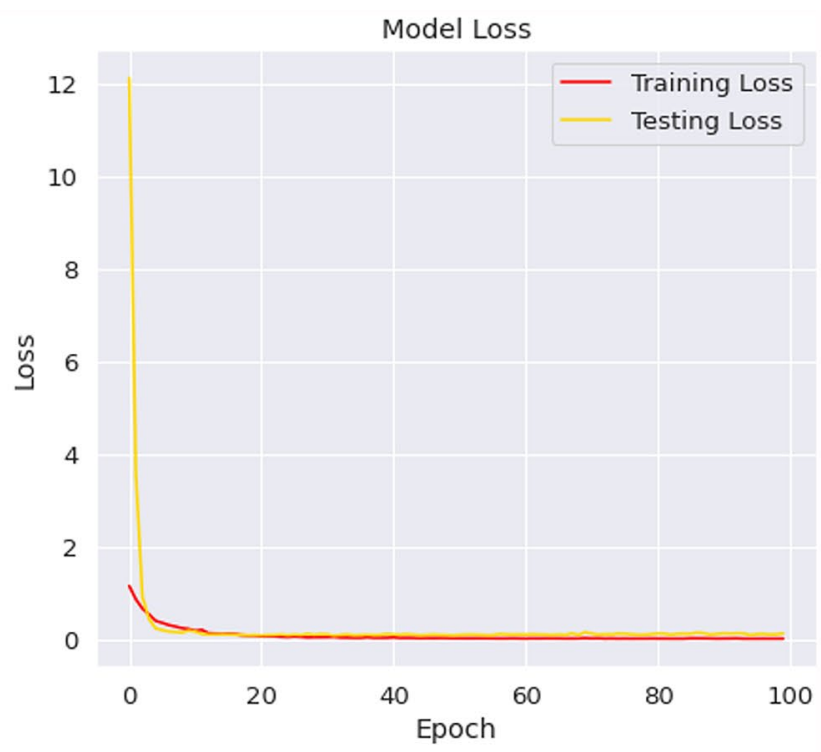

(b)

Fig. 9 Training and Testing, Fig. (a) Accuracy and Fig. (b) showing the Loss for multi-class experiment

\section{Conclusion and future scope}

The computerized systems for Covid-19 detection from Chest X-ray digital photographs can do binary and threeclass grouping without any human intervention with an accuracy of more than $90 \%$. Also, these systems are intelligent enough to work with larger and growing datasets. Furthermore, such systems play a vital role in such places where the test kits are not sufficiently available. Formerly, no significant acceptance from the exploration association of medicinal professionals for Covid disease case apprehension using X-ray digital images by deep learning and machine learning tools has been gained. With our proposed CheXImageNet model, the apex accuracies with other performance measures and top discrimination capacity are achieved by selecting the best layer combinations and parameters for training the deep convolutional neural network. Hence, for the initiatory judgment of Covid-19 disease, the proposed work can be a competent channel of examination for predicting Covid-19 disease in the human body and can help the radiologists and medical professionals.

Currently, we are progressing towards developing a dataset of digital images representing the Chest X-ray with an increasing number of digital images showing Covid-19 cases and also extending the technique for accomplishing much more robustness to make it used in detecting Covid-19 disease with both digital CT images and digital X-ray photographs. Over time, we intend to gather another Covid-19 disease patient's data and explore other possible up gradation together with categorization in added number of classes.
Also, in the future, current work can be extended by designing automated tools or software based on a multi-modality deep learning model using a large number of digital CT scans and X-ray images that must be evaluated by radiologists. For large data, neural networks may need to go deeper to learn more features to classify it correctly. Moreover, we elongate our work to use deep learning models to identify Covid-19 in a noisier environment.

Acknowledgements This research work is dedicated to Covid-19 frontline workers.

Funding This research did not receive any specific grant from funding agencies in the public, commercial, or not-for-profit sectors.

\section{Declarations}

Ethical approval This article does not contain any studies with human participants or animals performed by any of the authors.

Conflicts of interest The authors declare that they have no conflict of interest.

\section{References}

1. Huang C, Wang Y, Li X, Ren L, Zhao J, Hu Y, et al. Clinical features of patients infected with 2019 novel coronavirus in Wuhan, China. The Lancet. 2020;395(10223):497-506.

2. Shastri S, Singh K, Kumar S, Kour P, Mansotra V. Deep-LSTM ensemble framework to forecast Covid-19: an insight to the global pandemic. Int J Inf Technol. 1-11. 
3. WHO. Coronavirus disease 2019 (Covid-19) situation. 2020. https:// www.who.int/docs/default-source/coronaviruse/situation-reports/. Online. Accessed 12 April 2020.

4. Rio DC. Reverse transcription-polymerase chain reaction. Cold Spring Harb Protoc. 2014;2014(11):pdb-prot080887.

5. Punn NS, Sonbhadra SK, Agarwal S. COVID-19 Epidemic Analysis using Machine Learning and Deep Learning Algorithms. medRxiv. 2020.

6. Shastri S, Singh K, Kumar S, Kour P, Mansotra V. Time series forecasting of Covid-19 using deep learning models: IndiaUSA comparative case study. Chaos, Solitons \& Fractals. 2020;140:110227.

7. Wang W, Xu Y, Gao R, Lu R, Han K, Wu G, et al. Detection of SARS-CoV-2 in different types of clinical specimens. Jama. 2020;323(18):1843-4.

8. Xu X, Jiang X, Ma C, Du P, Li X, Lv S, et al. A deep learning system to screen novel coronavirus disease 2019 pneumonia. Engineering. 2020;6(10):1122-1129.

9. Zheng C, Deng X, Fu Q, Zhou Q, Feng J, Ma H, et al. Deep learning-based detection for COVID-19 from chest CT using weak label. MedRxiv. 2020.

10. Zhang J, Xie Y, Li Y, Shen C, Xia Y. Covid-19 screening on chest $\mathrm{X}$-ray images using deep learning based anomaly detection. arXiv preprint arXiv:200312338. 2020.

11. Jin YH, Cai L, Cheng ZS, Cheng H, Deng T, Fan YP, et al. A rapid advice guideline for the diagnosis and treatment of 2019 Novel Coronavirus (2019-nCoV) infected pneumonia (standard version). Mil Med Res. 2020;7(1):4.

12. Kansal I, Kasana SS. Fusion-based image de-fogging using dual tree complex wavelet transform. Int J Wavelets Multiresolution Inf Process. 2018;16(06):1850054.

13. Kansal I, Kasana SS. Improved color attenuation prior based image de-fogging technique. Multimed Tools Appl. 2020:1-23.

14. Furlow B. Radiation dose in computed tomography. Radiol Technol. 2010;81(5):437-50.

15. Huda W. Radiation doses and risks in chest computed tomography examinations. Proc Am Thorac Soc. 2007;4(4):316-20.

16. Rao ASS, Vazquez JA. Identification of COVID-19 can be quicker through artificial intelligence framework using a mobile phonebased survey when cities and towns are under quarantine. Infect Control Hosp Epidemiol. 2020;41(7):826-30.

17. Cheng JZ, Ni D, Chou YH, Qin J, Tiu CM, Chang YC, et al. Computer-aided diagnosis with deep learning architecture: applications to breast lesions in US images and pulmonary nodules in CT scans. Sci Rep. 2016;6(1):1-13.

18. Lakshmanaprabu S, Mohanty SN, Shankar K, Arunkumar N, Ramirez G. Optimal deep learning model for classification of lung cancer on CT images. Futur Gener Comput Syst. 2019;92:374-82.

19. Litjens G, Kooi T, Bejnordi BE, Setio AAA, Ciompi F, Ghafoorian $\mathrm{M}$, et al. A survey on deep learning in medical image analysis. Med Image Anal. 2017;42:60-88.

20. Zreik M, Lessmann N, van Hamersvelt RW, Wolterink JM, Voskuil M, Viergever MA, et al. Deep learning analysis of the myocardium in coronary $\mathrm{CT}$ angiography for identification of patients with functionally significant coronary artery stenosis. Med Image Anal. 2018;44:72-85.

21. Yang R, Li X, Liu H, Zhen Y, Zhang X, Xiong Q, et al. Chest CT severity score: an imaging tool for assessing severe COVID-19. Radiology: Cardiothoracic Imaging. 2020;2(2):e200047.

22. Wang S, Kang B, Ma J, Zeng X, Xiao M, Guo J, et al. A deep learning algorithm using CT images to screen for Corona Virus Disease (COVID-19). MedRxiv. 2020.
23. Wang L, Wong A. COVID-Net: a tailored deep convolutional neural network design for detection of COVID-19 cases from chest radiography images. 2020. https://arxiv.org/abs/2003.09871. Accessed; 2020.

24. Li L, Qin L, Xu Z, Yin Y, Wang X, Kong B, et al. Using artificial intelligence to detect COVID-19 and community-acquired pneumonia based on pulmonary CT: evaluation of the diagnostic accuracy. Radiology. 2020;296(2).

25. Ronneberger O, Fischer P, Brox T. U-net: Convolutional networks for biomedical image segmentation. In: International Conference on Medical image computing and computer-assisted intervention. Springer; 2015. p. 234-241.

26. Shibly KH, Dey SK, Islam MTU, Rahman MM. COVID Faster R-CNN: A Novel Framework to Diagnose Novel Coronavirus Disease (COVID-19) in X-Ray Images. medRxiv. 2020.

27. Li T, Han Z, Wei B, Zheng Y, Hong Y, Cong J. Robust Screening of COVID-19 from Chest X-ray via Discriminative Cost-Sensitive Learning. arXiv preprint arXiv:200412592. 2020.

28. Apostolopoulos ID, Mpesiana TA. Covid-19: automatic detection from $\mathrm{x}$-ray images utilizing transfer learning with convolutional neural networks. Physical and Engineering Sciences in Medicine. 2020;43(2):635-640.

29. Asif S, Wenhui Y. Automatic detection of COVID-19 using X-ray images with deep convolutional neural networks and machine learning. medRxiv. 2020.

30. Sethy PK, Behera SK, Ratha PK, Biswas P. Detection of coronavirus disease (COVID-19) based on deep features and support vector machine. 2020.

31. Tsiknakis N, Trivizakis E, Vassalou EE, Papadakis GZ, Spandidos DA, Tsatsakis A, et al. Interpretable artificial intelligence framework for COVID-19 screening on chest X-rays. Exp Ther Med. 2020;20(2):727-35.

32. Punn NS, Agarwal S. Automated diagnosis of COVID-19 with limited posteroanterior chest X-ray images using fine-tuned deep neural networks. arXiv preprint arXiv:200411676. 2020.

33. Huang Z, Liu X, Wang R, Zhang M, Zeng X, Liu J, et al. FaNet: fast assessment network for the novel coronavirus (COVID-19) pneumonia based on 3D CT imaging and clinical symptoms. Appl Intell. 2020:1-12.

34. Al-Bawi A, Al-Kaabi K, Jeryo M, Al-Fatlawi A. CCBlock: an effective use of deep learning for automatic diagnosis of COVID19 using X-ray images. Research on Biomedical Engineering. 2020:1-10.

35. Jamil M, Hussain I, et al. Automatic Detection of COVID-19 Infection from Chest X-ray using Deep Learning. medRxiv. 2020.

36. Makris A, Kontopoulos I, Tserpes K. COVID-19 detection from chest X-Ray images using Deep Learning and Convolutional Neural Networks. In: 11th Hellenic Conference on Artificial Intelligence. 2020. p. 60-66.

37. Ozturk T, Talo M, Yildirim EA, Baloglu UB, Yildirim O, Acharya UR. Automated detection of COVID-19 cases using deep neural networks with X-ray images. Comput Biol Med. 2020:103792.

38. Rahimzadeh M, Attar A. A modified deep convolutional neural network for detecting COVID-19 and pneumonia from chest X-ray images based on the concatenation of Xception and ResNet50V2. Informatics in Medicine Unlocked. 2020:100360.

Publisher's Note Springer Nature remains neutral with regard to jurisdictional claims in published maps and institutional affiliations. 